\title{
El cerebelo: su implicación en la dislexia
}

\author{
Angela Fawcett y Rod Nicolson
}

Departamento de Psicología, Universidad de Sheffield

UK

a.fawcett@sheffield.ac.uk 


\section{Resumen}

Introducción: En esta revisión exponemos, en líneas generales, el razonamiento y la evidencia que respalda nuestra hipótesis que los problemas sufridos por los disléxicos pueden atribuirse a un deficit cerebelar.

Método: Primero, ofrecemos una perspectiva general de la evidencia reciente que propone un papel importante del cerebelo en las habilidades cognitivas, particularmente las que apoyan el lenguaje hablado, además de su papel ya reconocido en las habilidades motrices. Segundo, resumimos la evidencia de nuestro laboratorio a favor de que la función cerebelar sea anormal en la dislexia.

Resultados: Consideramos dos líneas concretas de evidencia: la conductual, y la evidencia convergent de la neuroimagen, la cual demuestra diferencias significativas entre los grupos disléxicos y de control. También administramos la misma batería de pruebas conductuales a un grupo de niños que son malos lectores no discrepantes.

Discusión: Finalmente, proponemos una cadena causal ontogenético para el desarrollo de la dislexia como déficit cerebelar desde el nacimiento, considerando las implicaciones de este marco para las cuestiones clave en la investigación sobre la dislexia.

Palabras Clave: dislexia, cerebelo, fonología, cadena causal. 


\section{Introducción}

La dislexia evolutiva es el trastorno más extendido y más investigado de todos los trastornos evolutivos. La gran parte de la investigación de la última década ha considerado dos enfoques alternativos: la hipótesis del déficit fonológico y la hipótesis del déficit magnocelular. No obstante, a pesar de las investigaciones numerosas, estos enfoques no han convergido en un marco teórico aceptado. En nuestro laboratorio, hemos intentado discubrir las causas subyacentes de la dislexia, adoptando una perspectiva del aprendizaje. Defendemos que la lectura, a diferencia del lenguaje, no es una habilidad humana que haya evolucionado, y por lo tanto necesitamos comprender los procesos de aprendizaje para averiguar por qué los niños disléxicos no aprender a leer. La automatización no es un proceso consciente - a fuerza de práctica bajo condiciones más o menos constantes, la mayoría de los humanos simplemente 'adquieren' las habilidades--. Por lo tanto, la automatización explica de forma intuitivamente satisfactoria no sólo los problemas de lectura, sino también las dificultades fonológicas (pues la conciencia fonológica es una habilidad que se adquiere inicialmente sólo por escuchar el idioma de uno mismo). Lo que no quedó claro es POR QUÉ los niños disléxicos tienen problemas con la automatización de habilidades, y para esto buscamos una explicación a nivel del cerebro. Propusimos la hipótesis del déficit cerebelar (HDC) - que la anormalidad cerebelar era una causa de las dificultades que sufren los niños disléxicos-. En este artículo revisamos nuestro program de investigación a lo largo de quince años, y la evidencia para la HDC.

Hemos sostenido que un aspecto fascinante de la investigación sobre la dislexia se encuentra en que, sea lo que sea el interés del investigador - la lectura, la fonología, la escritura, la ortografía, la enseñanza, la memoria, la velocidad, la creatividad, el oído, el equilibrio, el aprendizaje, la habilidad, la genética, la estructura o la función del cerebro - los niños disléxicos mostrarán diferencias interesantes y poco comunes en ese dominio. Dada la necesidad de especializarse dentro de la ciencia, muchos investigadores han pasado a emprender estudios incisivos y penetrantes en su dominio específico de conocimiento. Esto explica cómo es que, por un lado, hay una profusión sin par de investigación sobre la dislexia, y que, por otro lado, la investigación no llega a acumularse, a empezar a construir una teoría global de la dislexia. Como la fábula hindí, a la que son muy aficionados los psicólogos, esto se parece a los cuatro ciegos que intentan describir un elefante. Uno toca el tronco, otro la pierna, otro la cola, y otro el costado, lo cual resulta en descripciones como "un tubo", "un árbol", "una casa", "y 
una soga". Si uno quiere describir el elefante como un todo, hace falta una variedad de perspectivas. Empecemos la recorrida del elefante identificando unas potentes causas de confusión en este terreno.

En estudios previos, Nicolson ha resumido las distintas motivaciones de los distintos investigadores dentro del Ecosistema de la Dislexia (Nicolson, 2002), las cuales nos conducen a un origen de confusión importante dentro de la investigación sobre la dislexia. Naturalmente, a muchos teóricos aplicados les interesa el rendimiento académico, sobre todo la alfabetización. Como consecuencia, analizan los distintos componentes de la lectura, investigan los efectos diferenciales de varias intervenciones, y con frecuencia subrayan (con razón) la necesidad de apoyo para cualquier niño que muestra riesgo de fracaso en la lectura, sea disléxico o no. Por contraste, los teóricos "puros" se interesan principalmente en las causes subyacentes de la dislexia (más que la alfabetización en sî) y por tanto emprenden pruebas motivadas teóricamente, con frecuencia dentro de esferas no relacionadas directamente con la alfabetización. En la mayoría de los campos de la ciencia, la diferencia entre la causa, los síntomas y el tratamiento está clara - por ejemplo, en la medicina, las causas, los síntomas, y el tratamiento de, digamos, de la malaria, son muy distintos-. De hecho, varias enfermedades pueden compartir síntomas parecidos. La gripe y la meningitis pueden dar lugar a síntomas de fiebre, dolores y náusea parecidos a los de la malaria; sin embargo las causas subyacentes (y el tratamiento) son muy distintos. En la dislexia, la diferencia queda mucho menos clara; por esto mismo es especialmente importante mantener las diferencias entre la causa, los síntomas y el tratamiento.

En estudios recientes, hemos comentado nuestra esperanza de que a lo largo de los próximos 5 años puedan identificarse varios sub-tipos de la dislexia, cada uno basado en una zona diferente del cerebro, pero que conduce cada uno a dificultades fonológicas básicas. Éstos pueden enlazarse con más síntomas y síntomas más distintivos (visuales, auditivos, motrices, dificultades de velocidad, etc.) de acuerdo con teorías actuales de la dislexia. Se han dado avances importantes en el estudio de coincidencias potenciales; volvemos a ello más adelante en este artículo. Los estudios que están en curso están descubriendo coincidencias entre tipos específicos de la dislexia y otros trastornos, incluyendo el ADHD, disfunción específica del lenguaje, dispraxia, y dificultad de aprendizaje generalizada. Esperamos que la identificación de causas subyacentes específicas pueda conducir a la especificación de las estrategias de intervención más apropiadas para un niño en concreto, además de aliviar los 
síntomas relativos a la lectura. Sobre todo, si se puede identificar una gama más amplia de precursores, podremos proporcionar apoyo activamente antes que los niños fracasen, y así romper el ciclo de fracaso para todo niño que tenga necesidades educativas especiales. El reto aplicado para los teóricos puros queda en esto.

Ya hemos expuesto los argumentos en favor de la investigación teórica pura, para que se identifiquen las causas subyacentes de la dislexia, pero, ¿qué haría falta para una teoría causal, y específicamente una teoría causal de la dislexia? Normalmente las explicaciones científicas pasan de las teorías descriptivas a las explicativas; éstas se basan en una buena descripción de lois síntomas, y la especificación de la base neurológica respectivamente. De modo parecido, Morton y Frith (1995) distinguen tres niveles de explicación - el biológico, el cognitivo y el conductual, el nivel biológico siendo el más profundo nivel explicativo-. Para nosotros, un marco adecuado para la dislexia responderá a las siguientes preguntas claves: ¿Cuál es la causa subyacente de la dislexia?; ¿Por qué parece ser algo específico de la lectura?; ¿Por qué las flaquezas parecen ser limitadas a la lectura?; y finalmente, dada la amplia gama de dificultades arriba enumeradas, ¿por qué existen tantas personas de alto rendimiento que son disléxicos?

Antes de dirigirnos a estas cuestiones, es importante considerar el papel creciente del cerebelo, el cual se ha pasado por alto, en gran medida, hasta hace poco.

\section{El Cerebelo}

El cerebelo es una estructura cerebral sub-córtica apretadamente atestada y profundamente doblada, situada en la parte de atrás del cerebro, a veces llamada el 'hind-brain' (Holmes, 1939). En los seres humanos, representa el 10-15\% del peso cerebral, el $40 \%$ del área superficial cerebral, y el 50\% de las neuronas del cerebro.

Los daños a distintas partes del cerebelo pueden llevar a distintos síntomas, los cuales incluyen los trastornos de la postura y el equilibrio, la rigidez de los miembros y la discoordinación o decomposición del movimiento (es decir, secuencias de movimientos anteriormente coordinadas, por ejemplo levantar una taza, pueden desglosarse en una serie de movimientos separados). Sin embargo, un aspecto del daño cerebelar es la gran plasticidad del sistema. 
Típicamente, un funcionamiento normal o casi normal se consigue nuevamente dentro de pocos meses del daño inicial (Holmes, 1922).

La propuesta implicación del cerebelo en las habilidades cognitivas ha llevado a una controversia considerable dentro del campo, pues el cerebelo tradicionalmente se ha considerado una zona motora (Eccles, Ito y Szentagothai, 1967; Holmes, 1917; Holmes, 1939; Stein y Glickstein, 1992), y también se le atribuye implicación en la automatización de habilidad motriz y en el control de aprendizaje adaptado a través de las estructuras cerebelares (Ito, 1984; Ito, 1990; Jenkins, Brooks, Nixon, Frackowiak y Passingham, 1994; Krupa, Thompson y Thompson, 1993). Sin embargo, como notan Leiner, Leiner y Dow (1989), ha evolucionado enormemente el cerebelo humano (en concreto, las hemisferas cerebelares laterales y el núcleo dentato cerebelar ventrolateral), enlazándose no sólo con las zonas motoras frontales, sino también con unas zonas de más adelante dentro de la corteza frontal, que incluyen la $\mathbf{D}^{-}$ na de lenguaje de Broca. Leiner, Leiner y Dow (Leiner et al., 1989; Leiner, Leiner y Dow, 1991; Leiner, Leiner y Dow, 1993) concluyeron que por tanto el cerebelo es fundamental para la adquisición de "destreza de lenguaje". En efecto, propusieron que el cerebelo está implicado de forma crítica en la automatización de cualquier habilidad, sea motriz o cognitiva. Queda controversia sobre el papel del cerebelo en las habilidades cognitivas que no implican el habla o el "habla interior" (Ackermann, Wildgruber, Daum y Grodd, 1998; Glickstein, 1993), pero ya ha evidencia abrumadora de la importancia del cerebelo en el lenguaje (Ackermann y Hertrich, 2000; Fabbro, Moretti y Bava, 2000; Silveri y Misciagna, 2000), en la percepción del habla (Mathiak, Hetrich, Grodd y Ackermann, 2002), hasta una demostración reciente de una implicación cerebelar específica en la lectura (Fulbright et al., 1999). Ahora incluso se ha demostrado que los pacientes con daño cerebelar muestran déficits en atención y en memoria de trabajo (Malm et al. 1998) y síntomas de tipo disléxico en la lectura (Moretti et al., 2002).

\section{La dislexia y el cerebelo}

Volvamos ahora a la dislexia, teniendo en cuenta la creciente coincidencia entre lo que se conoce de los déficits cerebelares y los síntomas de la dislexia. 


\section{El Programa de Investigación de Dislexia Sheffield}

En nuestro enfoque a la dislexia, el grupo Sheffield han sido poco usuales entre los investigadores de la dislexia, en que han adoptado una perspectiva de aprendizaje y de habilidades. El fuerte distintivo de la hipótesis de automatización fue que también era consecuente con los resultados de una serie a estudios a principios de los 1990, en los que investigamos un abanico de habilidades fuera del área de la alfabetización, y encontramos que nuestro panel de niños disléxicos mostró déficit graves en diversas habilidades. Éstas incluyeron el equilibrio (Fawcett y Nicolson, 1992; Nicolson y Fawcett, 1990; véase también Yap y van der Leij (1994)); la habilidad motriz (Fawcett y Nicolson, 1995b; véase también Daum et al. (1993)), procesamiento rápido (Fawcett y Nicolson, 1994; Nicolson y Fawcett, 1994). Además, considerando los datos como un conjunto (Nicolson y Fawcett, 1995a; Nicolson y Fawcett, 1995b), la mayoría de niños disléxicos (individuales) mostraban problemas generalizados, y no que distintos niños mostraban distintos perfiles, como se esperaría si hubiera una gama de sub-tipos (Boder, 1973; Castles y Holmes, 1996). El déficit de automatización por lo tanto aportó una explicación excelente de la gama de síntomas de la dislexia, pero no especificó una estructura neurológica subyacente. En investigaciones posteriores, esta hipótesis a "nivel cognitivo" se quedó absorbido dentro de la hipótesis a "nivel neurológico" del déficit cerebelar, como resumimos abajo.

\section{La hipótesis del déficit cerebelar}

Como hemos notado arriba, los déficits en habilidad motriz y en la automatización apuntan claramente al cerebelo. No obstante, los primeros hallazgos de Levinson (Frank y Levinson, 1973; Levinson, 1988) que defendían una ligera disfunción cerebelar fueron descartados en gran medida a causa de defectos en la metodología de investigación (Silver, 1987), relacionado con la creencia que el cerebelo no estaba implicado en las habilidades relacionadas con el lenguaje. Además, la hipótesis cae fuera del "supuesto de especificidad". Si de hecho existen problemas en el cerebelo, ¿cómo es que los síntomas principales son específicos al área de la lectura? 


\section{Método}

\section{Sujetos}

En nuestros intentos de responder a estas cuestiones, trabajos con nuestro panel de niños disléxicos "puros" con CI de más de 90, y edad lectora por lo menos 18 meses por detras de su edad cronológica, sin ninguna indicación de ADHD, y ningún problema importante a nivel emocional o conductual, y un grupo control de un trasfondo social similar, igualados en edad y CI. Los sujetos cumplieron los dos criterios excluyentes normales para la dislexia. Participaron tres grupos de niños disléxicos repartidos por edad, junto con tres grupos de íños de rendimiento normal. Se debe notar que los sujetos ya habían participado en varios experimentos, y que habíamos establecido que los sujetos con dislexia mostraron dificultades en habilidad fonológica, habilidad motriz, equilibrio y estimación temporal (Fawcett y Nicolson, 1995a; 1995b; Nicolson y Fawcett, 1994a; Nicolson, Fawcett y Dean, 1995). Los sujetos cobraron unos 5 euros por hora, y participaron con consentimiento plenamente informado.

\section{Pruebas clínicas de función cerebelar}

Los síntomas tradicionales de disfunción cerebelar con la distonia (problemas con el tono muscular) y la ataxia (trastorno de la postura, forma de andar, o movimientos de los miembros). Si hay daños cerebelares, los niños disléxicos deben mostrar estas señales tradicionales de la disfunción cerebelar (véase Holmes, 1917, 1939; y Dow y Moruzzi, 1958). Por consiguiente, reproducimos las pruebas descritas en Dow y Moruzzi (1958), con grupos de niños disléxicos y controles igualados de las edades de 18, 14 y 10 años (véase Fawcett, Nicolson y Dean, 1996). Las tareas eran de tres clases; postura y tono muscular; hipotonía de los miembros superiores; y movimiento voluntario complejo, un total de 14 tareas. La ejecución de los niños con dislexia fue significativamente peor que la de los controles de edad cronológica en todas las 14 tareas, y significativamente peor que los controles de edad lectora en 11 de las 14 pruebas. Utilizando "tamaño efectivo" apropiado a la edad en unidades de desviación típica (análogo a una puntuación de la -z) para cada prueba para cada niño (p.ej., Cohen, 1969), calculamos la gravedad y la incidencia de déficit en cada tarea. Los niños se les consideraron "de riesgo" si su ejecución fue inferior a la que se esperaba de su edad por una desviación típica o más, y los déficit de este tamaño o mayores se encontraron en todas las tareas menos una, varias de las tareas cerebelares mostrando déficit mayores que los de lectura. Estos resultados no se limitaron a los niños disléxicos de nuestro panel, sino se en- 
contraron también con una muestra adicional de 126 niños seleccionados de escuelas privadas especializadas en la dislexia. La muestra incluyó niños disléxicos y de control, con edades de 8 a 16, divididos en cuatro grupos por edad. Administramos tanto un abanico de tareas cerebelares y otras tareas sensibles a la dislexia. En todas las pruebas cerebelares, junto con las de segmentación y de repetición de no palabras, la ejecución de los niños disléxicos fue significativamente peor que la de los controles. Los análisis de tamaño efectivo también son parecidos al estudio del panel. De acuerdo con el estudio previo, comparando los niños disléxicos con los controles, algunos resultados más notables fueron la ejecución excepcionalmente mala de los cuatro grupos con dislexia en las tareas de estabilidad de postura y sacudida de miembros.

\section{Funcion cerebelar en el alumno lento}

Mucha investigación reciente desde los EEUU sugiere que no tiene sentido diferenciar entre los niños de baja CI y los niños con dislexia, ya que los dos muestran problemas con las habilidades fonológicas. Esta propuesta tiene implicaciones grandes en la investigación de la dislexia, y ha despertado controversia considerable en el campo: investigadores americanos como Stanovich (1988) y los Shaywitzes defienden que los grupos de malos lectores deben combinarse, e investigadores británicos, como Nicolson (1999) y Snowling et al. (2003), defienden que la distinción entre los grupos se debe mantener. Aunque por motivos diferentes, tanto Nicolson como Snowling, independientemente, defienden que una concentración en las habilidades fonológicas es demasiado limitada; Nicolas recomienda pruebas de habilidades más extensas, y Snowling recomienda pruebas más extensas de habilidades relacionadas con el lenguaje y la lectura. Para probar la hipótesis que merece la pena mantener la distinción entre niños disléxicos y niños lentos en la investigación, se administró una batería extensa de pruebas, incluyendo tareas fonológicas, de velocidad, motrices y cerebelares, a la cohorte entera de dos escuelas de niños con dificultades de aprendizaje. Se administraron las pruebas "a ciegas", sin acceder a los datos psicométricos sobre los niños. Después se colocaron los niños a un grupo de "discrepancia" según su CI, la mayoría $(n=29)$ se clasificaron como "nodiscrepantes' $(\mathrm{CI}<90)$ y un grupo más pequeño $(\mathrm{n}=7)$, con $\mathrm{CI}$ de al menos 90 , clasificados como 'discrepantes' (con dislexia). Los dos grupos mostraron déficits significativos relativo a los controles igualados por edad en casi todas las pruebas. En las tareas fonológicas, de velocidad y motrices, el grupo no-discrepante mostró disfunción al menos tan grave como el grupo discrepante. Por contraste, en las tareas cerebelares de estabilidad postural y do tono muscular, el grupo no discrepante las ejecutó significativamente mejor que los niños con dis- 
lexia, aproximándose al nivel de los controles. Los hallazgos indican que las pruebas cerebelares pueden ser un método valioso de diferenciar entre malos lectores con o sin discrepancia de CI.

El estudio que resumimos aquí pretendía establecer si los malos lectores con discrepancia de CI (niños con dislexia) se les puede distinguir de los malos lectores sin ninguna discrepancia (ML-SD), utilizando una gama de pruebas sobre habilidades que están establecidas como menos desarrolladas en los niños con dislexia. Se estableció una disociación entre los grupos con dislexia y los de ML-SD. En este estudio, las pruebas cerebelares se separaron en "estáticas" y "dinámicas", relacionadas con la distonia y la ataxia respectivamente. En esencia, las pruebas estáticas tenían que ver con la estabilidad y el tono muscular como respuesta a la perturbación del experimentador, mientras las pruebas dinámicas tenían que ver con la velocidad auto-generada de la ejecución de movimientos simples y complejos. Los niños de ML-SD ejecutaron las pruebas cerebelares estáticas a niveles casi normales, y eran significativamente mejores que los niños con dislexia en estas pruebas. Por contraste, en las pruebas dinámicas de velocidad, las pruebas fonológicas y de memoria verbal, y las pruebas de velocidad de procesamiento, los niños con ML-SD mostraron problemas iguales o significativamente mayores que los niños con dislexia. Los hallazgos proporcionan evidencia de la generalidad de déficits fonológicos y de velocidad tanto en los ML-SD como en los disléxicos, comparada con la especificidad de las pruebas cerebelares estáticas sobre el tono muscular y la estabilidad.

En conclusión, desde un punto de vista teórico, este estudio indica que hay diferencias entre los fenotipos de los niños con dislexia y los niños con dificultades de aprendizaje más generalizadas. Aunque bien podamos esperar cierta coincidencia entre los dos grupos, estos resultados indican que la mayoría de niños con dislexia sufren una anormalidad "cerebelar" ligera en las pruebas estáticas, mientras la mayoría de los niños de ML-SD no la sufren. Por supuesto, hay que reproducir estos resultados con otros grupos de niños con dislexia y grupos de ML-SD. La disociación entre las pruebas cerebelares y las pruebas fonológicas para estos grupos proporciona más apoyo firme para la hipótesis del déficit cerebelar (Nicolson, Fawcett y Dean, 1995). Además, sea lo que sea la interpretación específica que se haga, la disociación obtenida en el estudio presente entre niños de ML-SD y niños con dislexia demuestra que de hecho existen motivos teóricamente válidos para distinguir entre malos lectores con discrepancia y los malos lectores sin ella. 


\section{Pruebas directas de la anatomía y función cerebelares}

La falta de espacio impide que se incluyan un informe completo sobre la neuroanatomía del cerebelo (Finch et al, 2002), los estudios TEP (tomografía por emisión de positrones) sobre activación del cerebro en el aprendizaje motor (Nicolson et al, 1999), y el estudio del condicionamiento del parpadeo de ojos (Nicolson et al, 2002). Para una revisión de este trabajo, véase el debate de Trends in Neuroscience [Tendencias en la Neurociencia], en Nicolson et al, 2001. Lo más destacado procede del estudio TEP, donde, al ejecutar las tareas, los adultos disléxicos mostraron sólo un $10 \%$ del aumento de flujo sanguíneo en la corteza y vermis cerebelares que se encontró en los controles. Estos resultados son muy significativos, y no se predirían por medio de ninguna otra teoría de la dislexia. Proporcionan evidencia directa de que los indicios conductuales de anormalidad cerebelar sí reflejan anormalidades subyacentes en la activación cerebelar.

\section{Discusión}

Ahora consideremos las implicaciones de un déficit cerebelar para el conocimiento de la dislexia, de cómo se evoluciona la dislexia, y para trabajos futuros en este campo.

Empecemos por resumir la evidencia hoy por hoy. Nuestros estudios conductuales mostraron que un síntoma común de la ejecución de niños disléxicos es que no es tan bien automatizada, no sólo en las tareas de alfabetización sino en todas las demás tareas que se estudiaron. El papel establecido del cerebelo en el aprendizaje de habilidades y en la automatización lo recomendaba como buen candidato para la investigación, sobre todo cuando se considera juntamente con la evidencia de la neurociencia cognitiva sobre el papel fundamental del cerebelo en las tareas cognitivas relacionadas con el lenguaje. Demostramos que mostraba síntomas clínicos de anormalidad cerebelar no sólo nuestro panel de niños disléxicos, sino que también un grupo mucho más grande de niños disléxicos.

Pasamos a demostrar que merece la pena mantener la distinción entre niños con dislexia y niños con dificultades lectoras no-disléxicas, es decir, niños con dificultades más generalizadas. El mismo patrón de disfunciones se encontró en los dos grupos en cuanto a la fonología, la velocidad y las pruebas cerebelares dinámicas. Sin embargo, el grupo disléxico 
mostró déficits significativamente mayores en tareas cerebelares estáticas, las que implicaban la estabilidad postural y el tono muscular. Curiosamente, muchos grupos de investigadores adoptan una clasificación más amplia de la dislexia que la utilizada en Sheffield, incluyendo a niños con una CI de 80 o más, en contraste con el límite de 90 que se adoptó en las investigaciones de aquí. Esto significa que la interpretación de los resultados puede ser complicada por la presencia de malos lectores sin discreptancia dentro del grupo disléxico. Esto sugiere que los hallazgos de cualquier proyecto de investigación pueden ser significativamente distintos según los parámetros adoptados para la clasificación de grupos.

Nuestro estudio TEP sobre el aprendizaje de secuencias motrices mostró que, para los sujetos de nuestro panel, efectivamente existían anormalidades en la activación cerebelar en el procesamiento automático y en el nuevo aprendizaje. Ya que los sujetos disléxicos que habíamos revisado también mostraron señales clínicas clásicas del déficit cerebelar, esta demostración de que el grupo disléxico realmente tenía uso anormal del cerebelo, aportó a su vez mayor fuerza a nuestros hallazgos previos de que unos $80 \%$ de niños disléxicos muestran señales clínicas de anormalidad cerebelar.

Además, en la investigación aquí comentada, estamos empezando a acercarnos al bcus de los problemas dentro del cerebelo. La disociación entre pruebas estáticas y dinámicas para estos grupos ML-SD puede indicar que las anormalidades para los niños con dislexia se encuentran dentro de las partes laterales del lóbulo posterior del cerebelo, pues las lesiones a esta área suelen asociarse con la dismetria (movimiento inexacto de los miembros) y la hipotonía (tono muscular pobre) (Holmes, 1922). Estos hallazgos tienen particular interés a la vista de los hallazgos TEP recientes de patrones anormales de activación en el lóbulo posterior ipsilateral del cerebelo de adultos con dislexia, tanto al ejectuar una tarea de secuencia motriz previamente sobre-aprendida, como al aprender una nueva secuencia motriz (Nicolson et al., 1999).

\section{Hacia una explicación causal}

Ahora podemos empezar a rellenar los blancos de una cadena causal ontogenética. Fíjese en el impacto de estas dificultades de aprendizaje en la memoria de trabajo, la cual claramente está afectada en la dislexia. 


\section{Figura 1 La dislexia: una cadena causal ontogenética}

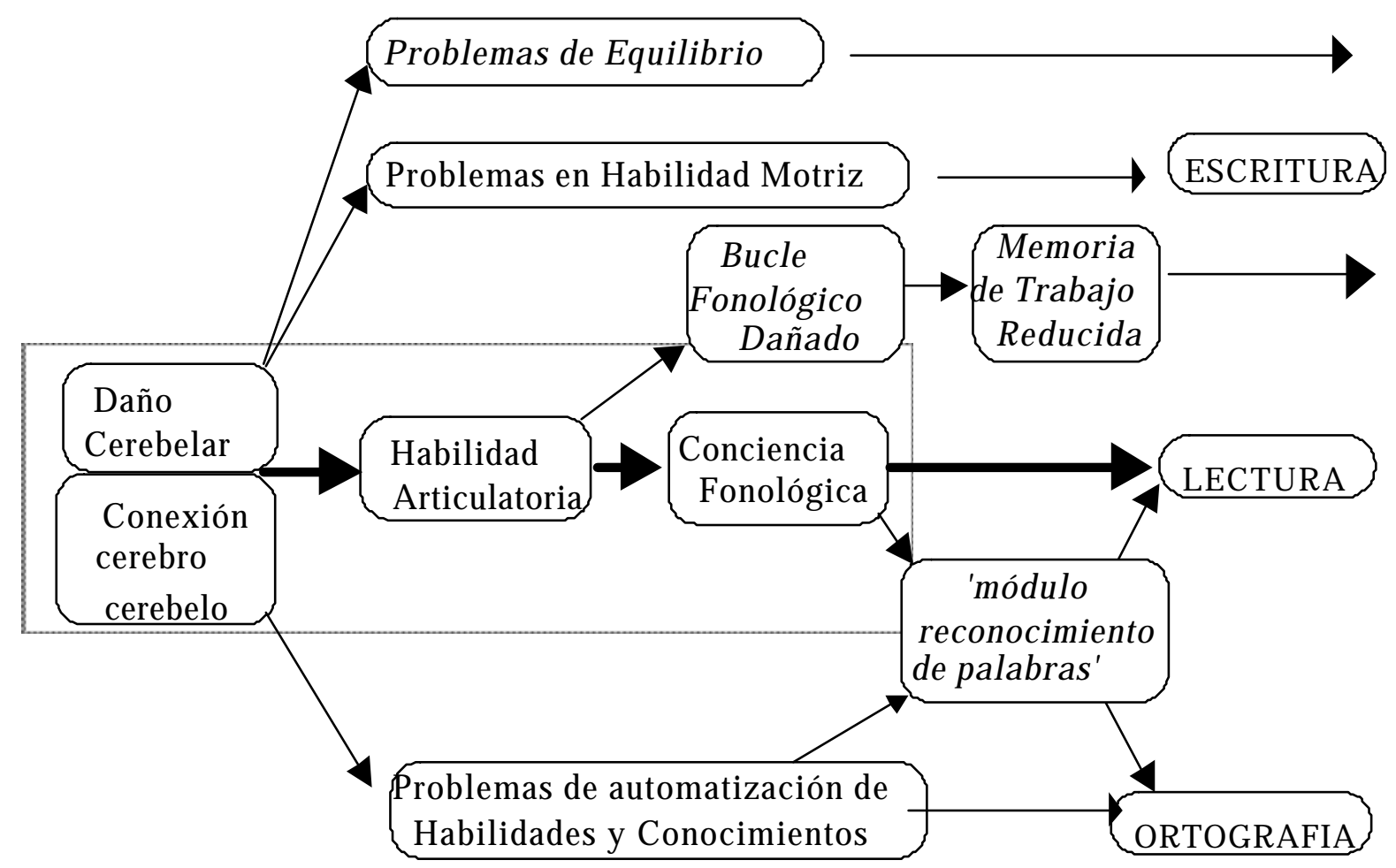

En la Figura 1 (de Nicolson y Fawcett, 1999), se traza la cadena causal ontogenética hipotético entre problemas cerebelares, dificultades fonológicas, y los consiguientes problemas lectoras, dando cuenta de las tres dificultades de criterio: la escritura, la lectura y la ortografía. Con frecuencia los niños disléxicos tienen letra de pobre calidad, lo que ha sido difícil de explicar bajo otras teorías, pero que sí puede tratar con naturalidad la HDC, como habilidad motriz que requiere un control temporal exacto y coordinación de los músculos. Aunque las dificultades de alfabetización surgen por varias rutas, la ruta céntrica se subraya como la más importante. Si un bebé tiene disfunción cerebelar, ésta se verá primero como una ligera dificultad motriz - el bebé puede tardar más para incorporarse y para andar, y puede que tenga problemas mayores con el control muscular fino-. Estos problemas pueden parecer no muy graves, a menos que apreciemos que nuestra habilidad motriz más compleja es la articulación, y, por consiguiente, el bebé puede tardar más para empezar a balbucear (véase p.ej., Ejiri y Mastaka, 2001 para evidencia que relaciona el balbuceo con el control motriz), y, más adelante, para hablar (cfr. Bates y Dick, 2002). Una vez desarrollados el habla y el andar, las habilidades pueden ser menos fluidas, menos "diestras", en los niños con disfunción cerebelar. Si la articulación es menos fluida que lo normal, ocupa más recursos conscientes, dejando menos recursos para procesar la retroalimentación sensorial. El procesamiento de la estructura auditiva y fonémica de las palabras habladas puede ser menos completo, llevando a la falta de 
conciencia de inicio, rima y la estructura fonémica del lenguage (véase Snowling y Hulme, 1994). Por tanto, se predice que la disfunción cerebelar causaría el "déficit fonológico" básico que se ha mostrado tan provechoso como marco explicativo para la dislexia. Básandose en este marco, las explicaciones normales de los déficit lectores son vigentes, junto con los problemas de aprendizaje y de automatización, los que llevan a menos fluidez y menos velocidad en la lectura, o sea, la hipótesis del doble déficit (Wolf y Bowers, 1999). Una de las claves de la lectura fluida es la capacidad de articular a nivel subvocal, y se sabe que el cerebelo está activado en el habla interno (Thach, 1996). La tercera habilidad de criterio, o sea, la ortografía, puede ser la que más resiste la rehabilitación, debido a la combinación de una lectura que cuesta mucho esfuerzo, una pobre conciencia fonológica, y dificultades en las habilidades de automatización y la eliminación de errores, además del uso simultáneo de habilidades tanto fonológicas como motrices (Thomson, 1984).

Esto nos lleva otra vez a las cuestiones claves de la investigación sobre la dislexia. Ya hemos comentado el mecanismo y la dirección de la causalidad, y sugerimos que las dificultades son específicas a la lectura y a la ortografía porque éstas implican una combinación de habilidades fonológicas, fluidez, automatización y la realización de varias tareas a la vez una combinación de todas las habilidades que son difíciles para los niños disléxicos. ¿Cómo es que la ejecución de otras habilidades parece ser normal? Dado que la alfabetización es de tan crítica importancia educativa, se le examina minuciosamente, mientras otras habilidades en gran medida se les pasa por alto. Por otra parte, muchas habilidades no tienen disfunción, o incluso están sobrecompensadas, pues se puede adquirir habilidades sin mucha implicación del cerebelo, simplemente requiere más implicación "frontal" - precisamente el patrón que se encuentra en nuestra tarea de aprendizaje de una secuencia-. La falta de automaticidad solamente llega a ser un problema real si se requiere procesamiento rápido o la realización de varias tareas a la vez, porque la mayoría de las habilidades incluyen habilidades "intelectuales" que requieren implicación frontal - el pensamiento en lugar de la memorización-. Esto nos conduce de nuevo a una explicación para la discrepancia entre el pobre rendimiento lector y el buen funcionamiento intelectual de los niños con dislexia. Existe evidencia que los adultos con dislexia pueden estar entre los más creativos y más triunfadores de su generación (West, 1991). ¿Cómo se puede explicar esto a la luz de la disfunción cerebelar que aparentemente produce dificultades importantes en la adquisición de habilidades, y con la habilidad lingüística? La capacidad de razonamiento no depende de la fluidez. De hecho, bien puede que la fluidez sea el enemigo de la creatividad - el intento de resolver nuevas clases de problemas 
que requieren una manera distinta de pensar sobre el problema y sobre sus elementos - pues la fluidez en esencia consiste en la capacidad de repetir acciones o pensamientos previos cada vez más rápidamente sin pensamiento consciente.

\section{Interpretaciones según las hipótesis alternativas}

Hemos sugerido que el marco HDC absorbe de manera natural la hipótesis fonológica, y que la hipótesis del doble déficit (Wolf y Bowers, 1999) se puede explicar de igual manera, ya que la hipótesis del doble déficit es una descripción a nivel cognitivo. Sin embargo, queda la pregunta clave: ¿Por qué los niños se vuelven más rápidos al madurar? Parece probable que esto refleje una eficacia mejorada de los mecanismos fundamentales del procesamiento, en los que se implicará de manera fundamental el cerebelo.

Stein (p.ej., Stein y Walsh, 1997) sostiene que la disfunción cerebelar se le puede atribuir a un input defectuoso por medio de unos caminos magnocelulares defectuosos. Parece claro que existe un sub-tipo de la dislexia con disfunción magnocelular, y Tallal (Tallal, Miller y Fitch, 1993) ha sugerido que puede haber disfunción sensorial general, que incluye tanto el rendimiento motriz como la recepción visual y auditiva. Stein nota que hay magnocélulas en el cerebelo y en los sistemas de rendimiento motriz, lo cual hace más difícil distinguir estas teorías de la HDC. No obstante, si uno limita la hipótesis del déficit magnocelular a la fase de recepción sensorial, no queda claro por qué los niños disléxicos tienen dificultad en la detección de rimas, que no implican el procesamiento rápido. Partiendo de nuestro propio trabajo, no hay ninguna explicación magnocelular obvia para la velocidad normal de reacciones simples, cuando la misma respuesta retrasa si hay que hacer una elección (Nicolson y Fawcett, 1994); tampoco se explica las dificultades en estimacioness temporales, el bajo tono muscular o la falta de activación cerebelar anormal en la tarea de prendizaje de una secuencia motriz. Puede que las investigaciones futuras descubran un subtipo "magnocelular", un subtipo "cerebelar", y varios subtipos "mixtos".

\section{Las investigaciones más generales sobre el cerebelo y la dislexia}

Posteriormente a la primera presentación del la HDC, el papel del cerebelo en la lectura y en los procesos cognitivas ha pasado de la controversia a la ortodoxia, los investigadores ya intentando localizar las zonas del cerebelo que se implican en el lenguaje. Los hallazgos 
recientes incluyen la activación del cerebelo en operaciones mentales no motrices (Hanakawa et al, 2002) o incluso la generación de antónimos, en la ausencia de movimiento mental (Gebhart et al, 2002). Un meta-analysis (Turkeltaub et al., 2002) concluyó que en la lectura en voz alta de palabras sueltas, el cerebelo se activaba de forma consecuente. Sin embargo, en un meta-análisis de 35 estudios de neuroimagen de la doble ruta de la lectura, se encontró que raramente se comentaban zonas como el cerebelo, aunque se encontraban activadas de forma consecuente (Jobard, Crivello y Tzourio-Mazoyer, 2003 en prensa). En lo que fue quizá lo más ilusionante para la hipótesis del déficit cerebelar, Bower y Parsons (2003) utilizaron su investigación sobre el tacto para sacar una nueva hipótesis sobre el papel del cerebelo en el procesamiento sensorial.

Investigaciones recientes sobre la dislexia y procedentes de otros grupos han estudiado tanto el déficit cerebelar como el déficit de procesamiento sensorial. El grupo de Stein comparó el metabolismo del cerebelo de los disléxicos con el de los controles, utilizando el electroscopio de resonancia magnética. Encontraron una proporción menor en el cerebelo de los disléxicos, comparada con los controles, sobre todo por el lado derecho (Rae et al. 1998). Más recientemente, otro estudio de Rae et al. (2002) utilizó las imágenes para mostrar simetría cerebelar en los disléxicos pero no en los controles. Rae et al. argumentaron que la relación de la asimetría cerebelar con la habilidad de descodificación fonológica y con el ser diestro o zurdo, junto con su hallazgo previo de proporciones cambiadas de metabolitos en el cerebelo de los disléxicos, sugieren que hay cambios en la organización neurológica del cerebelo que se relacionan con las habilidades de descodificación fonológica, además de las habilidades motrices y el ser diestro o zurdo.

Se ha publicado evidencia de otros países, como Noruega, sobre la incidencia de déficits de equilibrio y de la forma de andar, relacionándola con el cerebelo en los niños con dislexia (Moe-Nilssen et al, 2003), y midiendo más precisamente la postura a través de un acelerómetro. De forma parecida, se ha realizado una serie de estudios utilizando el polhemus, un aparato para medir la posición de los miembros en un espacio de 3 dimensiones, con resultados preliminares que indican diferencias significativas entre los disléxicos y los controles. Estudios en Italia sobre el aprendizaje implícito han identificado déficits en los niños disléxicos que se han relacionado con el cerebelo (Vicari et al, 2003). Moores et al. (2003) del laboratorio Sheffield, han sido los más notables en estudiar la atención, además de Facoetti et al. (2003). En cuanto a la activación del cerebro, las imágenes dejaron ver el lóbulo anterior 
derecho significativamente más pequeño en los sujetos disléxicos, correlacionado con déficits en la lectura, la ortografía y el lenguaje, los que se asocian a la dislexia. Además, los individuos con dislexia se les podía distinguir de los controles por el volumen del lóbulo anterior derecho del cerebelo (Eckert et al, 2002). Por otra parte, se ha realizado una serie de estudios del condicionamiento del parpadeo del ojo (el cual se conoce que es mediado por el cerebelo) que muestran disfunciones en la dislexia (Coffin et al, 2003). Todos estos estudios proporcionan apoyo firme para la hipótesis del déficit cerebelar de la dislexia.

Sin embargo, se han realizado estudios del déficit cerebelar que han llegado a conclusiones menos positivas, incluyendo el trabajo de Wimmer (Wimmer el al, 1999), que concluye que los niños con DDAH son los más afectados en las habilidades cerebelares. No obstante, es probable que los disléxicos de habla alemana sean diferentes que los disléxicos ingleses, porque muestran sólo déficits de velocidad, no de precisión. Sigue adelante el trabajo con niños con DDAH, pero actualmente hay escasez de trabajos publicados que estudian los niños con distintos diagnósticos a partir de la misma batería de pruebas. Los trabajos de Ramus y compañeros han identificado diferencias significativas entre los niños disléxicos y controles en el equilibrio y en otras tareas cerebelares, aunque no se encontraron diferencias significativas en las pruebas de estimación temporal (Ramus et al., 2003a y b).

\section{Conclusiones}

Tanto virtud como limitación de las investigaciones de Sheffield es el que hayamos trabajado con niños disléxicos "puros", adrede excluyendo a los niños que estaban en el límite de ser disléxicos, o niños comórbidos de DDAH/dislexia. Incluimos todos los niños disléxicos que cumplieron nuestros criterios (y que estaban dispuestos a participar), así evitando alguna predisposición de la misma selección. Sin embargo, esto supuso que los grupos eran pequeños y los resultados quizás no generalicen del todo a otros grupos. Es una clara prioridad establecer la difusión de síntomas cerebelares en poblaciones más grandes de niños disléxicos (y grupos comórbidos).

Nos es grato notar que estas investigaciones ya están en curso, el grupo Sheffield está trabajando con niños con dificultades de aprendizaje generalizadas, DDAH y dispraxia, igual que la dislexia, y otros grupos de investigación estudian aspectos más amplios del rendimiento de niños con un abanico de dificultades. 
En conclusión, la hipótesis del déficit cerebelar es una hipótesis a nivel biológico que está bien descrita a nivel cognitivo como una hipótesis del déficit de automatización. Las dos hipótesis en conjunto han proporcionado una explicación causal verdadera de los diversos hallazgos de la investigación de la dislexia. Sin duda las futuras investigaciones mostrarán que el cuadro todavía no está completo, pero mientras tanto la HDC genera varias vías nuevas e interesantes por las que investigar la dislexia. Una dirección estimulante donde seguir investigando es la disociación entre los hallazgos para malos lectores disléxicos y los no discrepantes en las pruebas cerebelares estáticas y dinámicas, lo cual puede representar el comienzo de la resolución de uno de los aspectos más controvertidos en la investigación de la dislexia.

\section{Referencias}

Ackermann, H. y Hertrich, I. (2000). The contribution of the cerebellum to speech processing. Journal of Neurolinguistics, 13(2-3), 95-116.

Ackermann, H., Wildgruber, D., Daum, I., y Grodd, W. (1998). Does the cerebellum contribute to cognitive aspects of speech production? A functional magnetic resonance imaging (fMRI) study in humans. Neuroscience Letters, 247, 187-190.

Bates, E., y Dick, F. (2002). Language, gesture, and the developing brain. Developmental Psychobiology, 40(3), 293-310

Bower, J.M. y Parsons, L.M. (2003). Rethinking the lesser brain. Scientific American, 289,5057.

Coffin, J. M., Baroody, S., Schneider, K y O’Neill, J. (2003 en prensa). Impaired Cerebellar Learning In Children With Prenatal Alcohol Exposure: A Comparative Study of Eyeblink Conditioning In Children with ADHD Cortex, en prensa.

Cohen, J. (1969). Statistical power analysis for the behavioural sciences. New York: Academic Press.

Daum I., Schugens M., Ackermann H., Lutzenberger W., Dichgans J., y Birbaumer N. (1993) Classical Conditioning after cerebellar lesions in humans. Behavioral Neuroscience 107, 748-756

Dow, R. S., \& Moruzzi, G. (1958). The physiology and pathology of the cerebellum. Minneapolis: University of Minnesota Press. 
Eckert, M. A., Leonard, C. M., Richards, T., Aylward, E., Thomson, J., y Berninger, V. (2002, en prensa). Anatomical Correlates of Dyslexia: Frontal and Cerebellar Findings. Brain.

Eccles, J. C., Ito, M., y Szentagothai, J. (1967). The cerebellum as a neuronal machine. New York: Springer-Verlag.

Ejiri, K., y Masataka, N. (2001). Co-occurrence of preverbal vocal behavior and motor action in early infancy. Developmental Science, 4(1), 40-48.

Fabbro, F., Moretti, R., y Bava, A. (2000). Language impairments in patients with cerebellar lesions. Journal of Neurolinguistics, 13(2-3), 173-188.

Facoetti, A., Turatto, M., Lorusso, M. L., Mascetti, G.G., (2001). Orienting of visual attention in dyslexia: evidence for asymmetric hemispheric control of attention. Experimental Brain Research 138, 46-53

Facoetti, A., Lorusso, M. L., Paganoni, P., Cattaneo, C., Galli, R., Umilta, C., y Mascetti, G. G. (2003). Auditory and visual automatic attention deficits in developmental dyslexia. Brain Res Cogn Brain Res, 16(2), 185-191

Fawcett, A. J., y Nicolson, R. I. (1994). Naming speed in children with dyslexia. Journal of Learning Disabilities, 27, 641-646.

Fawcett, A. J., y Nicolson, R. I. (1995a). Persistence of phonological awareness deficits in older children with dyslexia. Reading and Writing, 7, 361-376.

Fawcett, A. J. y Nicolson, R. I. (1995b). Persistent deficits in motor skill for children with dyslexia. Journal of Motor Behaviour, 27, 235-240

Fawcett, A. J., y Nicolson, R. I. (1999). Performance of dyslexic children on cerebellar and cognitive tests. Journal of Motor Behaviour, 31, 68-78.

Fawcett, A. J., Nicolson, R. I., y Dean, P. (1996). Impaired performance of children with dyslexia on a range of cerebellar tasks. Annals of Dyslexia, 46, 259-283.

Finch, A. J., Nicolson, R. I., y Fawcett, A. J. (2002). Evidence for a neuroanatomical difference within the olivo-cerebellar pathway of adults with dyslexia. Cortex, 38(4), 529539.

Frank, J., y Levinson, H. N. (1973). Dysmetric dyslexia and dyspraxia: hypothesis and study. Journal of American Academy of Child Psychiatry, 12, 690-701.

Fulbright, R. K., Jenner, A. R., Mencl, W. E., Pugh, K. R., Shaywitz, B. A., Shaywitz, S. E., Frost, S. J., Skudlarski, P., Constable, R. T., Lacadie, C. M., Marchione, K. E., y Gore, J. C. (1999). The cerebellum's role in reading: A functional MR imaging study. American Journal of Neuroradiology, 20, 1925-1930. 
Gebhart, A. L., Petersen, S.E. y Thach, W. T., (2002). Role of the posterolateral cerebellum in language. En Highstein y Thach (Eds.). The Cerebellum: Recent developments in cerebellar research: Special Issue of the Annals of the New York Academy of Sciences), 978, 318-333.

Glickstein, M. (1993). Motor skills but not cognitive tasks. Trends in Neuroscience, 16, 450451.

Hanakawa, T., Honda, M., Sawamoto, N., Okada, T., Yonekura, Y., Fukuyama, H., y Shibasaki, H. (2002). The role of Rostral Brodmann Area 6 in Mental Operation tasks: an integrative neuroimaging approach. Cerebral Cortex, 12, 1157-1170.

Holmes, G. (1917). The symptoms of acute cerebellar injuries due to gunshot injuries. Brain, 40, 461-535.

Holmes, G. (1922). Clinical symptoms of cerebellar disease and their interpretation. Lancet, $1,1177-1237$.

Holmes, G. (1939). The cerebellum of man. Brain, 62, 1-30.

Ito, M. (1984). The cerebellum and neural control. New York: Raven Press.

Ito, M. (1990). A new physiological concept on cerebellum. Revue Neurologique (Paris), 146, 564-569.

Ivry, R. B., y Keele, S. W. (1989). Timing functions of the cerebellum. Journal of Cognitive Neuroscience, 1, 136-152.

Jenkins, I. H., Brooks, D. J., Nixon, P. D., Frackowiak, R. S. J., y Passingham, R. E. (1994). Motor sequence learning - a study with Positron Emission Tomography. Journal of Neuroscience, 14, 3775-90.

Jobard, J., Crivello F., y Tzourio-Mazoyer, N (2003). Evaluation of the dual route theory of reading: a metanalysis of 35 neuroimaging studies. Neuroimage en prensa

Krupa, D. J., Thompson, J. K., y Thompson, R. F. (1993). Localization of a memory trace in the mammalian brain. Science, 260, 989-991.

Leiner, H. C., Leiner, A. L., y Dow, R. S. (1989). Reappraising the cerebellum: what does the hindbrain contribute to the forebrain. Behavioural Neuroscience, 103, 998-1008.

Leiner, H. C., Leiner, A. L., y Dow, R. S. (1991). The human cerebro-cerebellar system: Its computing, cognitive, and language skills. Behav Brain Res, 44, 113 - 128.

Leiner, H. C., Leiner, A. L., y Dow, R. S. (1993). Cognitive and language functions of the human cerebellum. Trends in Neuroscience, 16, 444-447.

Levinson, H. N. (1988). The cerebellar-vestibular basis of learning disabilities in children, adolescents and adults: Hypothesis and study. American Psychiatric Association Annual 
Meeting New Research Session (1987, Chicago, Illinois). Perceptual and Motor Skills, 67(3), 983-1006.

Levinson, H. N. (1990). The diagnostic value of cerebellar-vestibular tests in detecting learning disabilities, dyslexia, and Attention Deficit Disorder. Perceptual and Motor Skills, 71(1), 67-82.

Malm, J., Kristensen, B., Karlsson, T., Carlberg, B., Fagerlund, M., y Olsson, T. Cognitive impairment in young adults with infratentorial infarcts. Neurology, 51, 433-440, 1998.

Mathiak, K., Hetrich, I., Grodd W, y Ackermann, H. (2002), Cerebellum and speech perception: a functional magnetic resonance imaging study. Journal Cognitive Neuroscience, $14,902-912$

Moe-Nilssen R., Helbostad J.L., Talcott J.B., Toennessen F.E. (2003) Balance and gait in children with dyslexia. Experimental Brain Research, 150, 2237-244

Moores, E., Nicolson, R., y Fawcett, A. J. (2003). Attention deficits in dyslexia: evidence for an automatisation deficit? European Journal of Cognitive Psychology, 15(3), 321-348

Moretti, R., Bava, A., Torre, P., Antonello, R. M., y Cazzato, G. (2002). Reading errors in patients with cerebellar vermis lesions. J Neurol, 249(4), 461-468.

Morrison, F. J., y Manis, F. R. (1983). Cognitive processes in reading disability: a critique and proposal. En C. J. Brainerd y M. Pressley (Eds.), Progress in Cognitive Development Research, . New York: Springer-Verlag.

Morton, J., y Frith, U. (1995). Causal modelling: A structural approach to developmental psychopathology. En D. Cicchetti \& C.D.J. (Eds.), Manual of Developmental Psychopathology (vol. 2, pp. 274-298. New York: Wiley.

Nicolson, R. I., y Fawcett, A. J. (1990). Automaticity: A new framework for dyslexia research? Cognition, 35(2), 159-182.

Nicolson, R. I., y Fawcett, A. J. (1994). Reaction Times and Dyslexia. Quarterly Journal of Experimental Psychology, 47A, 29-48.

Nicolson, R. I., y Fawcett, A. J. (1995a). Balance, phonological skill and dyslexia: Towards the Dyslexia Early Screening Test. Dyslexia Review, 7, 8-11.

Nicolson, R. I., y Fawcett, A. J. (1995b). Dyslexia is more than a phonological disability. Dyslexia: An international journal of research and practice, 1, 19-37.

Nicolson, R. I., y Fawcett, A. J. (1999). Developmental Dyslexia: The role of the cerebellum. Dyslexia: An International Journal of Research and Practice, 5, 155-177. 
Nicolson, R. I., Fawcett, A. J., y Dean, P. (1995). Time-estimation deficits in developmental dyslexia - evidence for cerebellar involvement. Proceedings of the Royal Society of London Series B-Biological Sciences, 259, 43-47.

Passingham, R. E. (1975). Changes in the size and organization of the brain in man and his ancestors. Brain Behaviour and Evolution, 11, 73-90.

Rae, C., Lee, M. A., Dixon, R. M., Blamire, A. M., Thompson, C. H., Styles, P., Talcott, J., Richardson, A. J., \& Stein, J. F. (1998). Metabolic abnormalities in developmental dyslexia detected by H-1 magnetic resonance spectroscopy. Lancet, 351, 1849-1852.

Rae, C., Harasty, J. A., Dzendrowskyj, T. E., Talcott, J. B., Simpson, J. M., Blamire, A. M., Dixon, R. M., Lee, M. A., Thompson, C. H., Styles, P., Richardson, A. J., y Stein, J.F. (2002). Cerebellar morphology in developmental dyslexia. Neuropsychologia, 40(8), 1285-1292.

Ramus, F, Pidgeon, E., y Frith, U. (2003a) The relationship between motor control and phonology in dyslexic children. Journal of Child Psychology and Psychiatry and dlied disciplines, 54, 712-722.

Ramus, F. (2003b). Developmental dyslexia: specific phonological deficit or general sensorimotor dysfunction? Current Opinion in Neurobiology, 13, 212-218

Seidenberg, M. S. (1993). Connectionist models and cognitive theory. Psychological Science, 4, 228-235.

Silver, L. B. (1987). The 'magic cure': A review of the current controversial approaches for treating learning disabilities. Journal of Learning Disabilities, 20, 498-505.

Silveri, M. C., y Misciagna, S. (2000). Language, memory, and the cerebellum. Journal of Neurolinguistics, 13(2-3), 129-143.

Snowling, M., J, Gallagher, A., Frith, U. (2003). Family risk of dyslexia is continuous: Individual differences in the precursors of reading skill. Child development, 74 (2): 358373.

Snowling, M., y Hulme, C. (1994). The development of phonological skills. Philosophical Transactions of the Royal Society of London Series B-Biological Sciences, 346, 21-27.

Stanovich, K. E. (1988). Explaining the differences between the dyslexic and the gardenvariety poor reader: The phonological-core variable-difference model. Journal of Learning Disabilities, 21, 590-612.

Stein, J., y Walsh, V. (1997). To see but not to read; The magnocellular theory of dyslexia. Trends in Neurosciences, 20, 147-152. 
Stein, J. F., y Glickstein, M. (1992). Role of the cerebellum in visual guidance of movement. Physiological Reviews, 72, 972-1017.

Tallal, P., Miller, S., y Fitch, R. H. (1993). Neurobiological Basis of Speech: A Case for the Preeminence of Temporal Processing. Annals of the New York Academy of Sciences, $682,27-47$.

Thach, W. T. (1996). On the specific role of the cerebellum in motor learning and cognition: Clues from PET activation and lesion studies in man. Behavioural and Brain Sciences, 19, 411-431.

Thomson, M, (1984). Developmental dyslexia: Its nature, assessment and remediation. London: Edward Arnold.

Turkeltaub, P. E., Eden, G. F. Jones, K. M. y Zeffiro, T. A. (2002) Meta-analysis of the functional neuroanatomy of single-word reading: Method and validation. Neuroimage 16, 765-780.

Vellutino, F. R. (1979). Dyslexia: Theory and research. Cambridge, MA: MIT Press.

Vicari, S., Marotta, L., Menghini, D., Molinari, M., \& Petrosini, L. (2003). Implicit learning deficit in children with developmental dyslexia. Neuropsychologia, 41(1), 108-114

Wimmer H., Mayringer H., y Raberger T. (1999) Reading and dual-task balancing: Evidence against the automatization deficit explanation of developmental dyslexia Journal of Learning Disabilities, 32, 473-478

West, T. G. (1991). In the Mind's Eye: Visual thinkers, gifted people with learning difficulties, computer images, and the ironies of creativity. Buffalo NY: Prometheus Books.

Wolf, M., y Bowers, P. G. (1999). The double-deficit hypothesis for the developmental dyslexias. Journal of Educational Psychology, 91, 415-438. 
El cerebelo: su implicación en la dislexia

[Esta página está dejada en blanco intencionadamente] 